Astronomy Letters, Vol. 30, No. 11, 2004, pp. 785-796.

Translated from Pis'ma v Astronomicheski Zhurnal, Vol. 30, No. 11, 2004, pp. 861-873.

\title{
KINEMATIC PECULIARITIES OF GOULD BELT STARS
}

\author{
V. V. Bobylev \\ Pulkovo Astronomical Observatory, Russian Academy of Sciences, \\ Pulkovskoe shosse 65, St. Petersburg, 196140 Russia \\ E-mail:vbobylev@gao.spb.ru
}

Received November 12, 2004

\begin{abstract}
We analyzed the space velocities of Gould Belt stars younger than $125 \mathrm{Myr}$ located at heliocentric distances $<650 \mathrm{pc}$. We determined the rotation and expansion parameters of the Gould Belt by assuming the existence of a single kinematic center whose direction was found to be the following: $l_{\circ}=128^{\circ}$ and $R_{\circ}=150 \mathrm{pc}$. The linear velocities reach their maximum at a distance of $\approx 300 \mathrm{pc}$ from the center and are $-6 \mathrm{~km} \mathrm{~s}^{-1}$ for the rotation (whose direction coincides with the Galactic rotation) and $+4 \mathrm{~km} \mathrm{~s}^{-1}$ for the expansion. The stellar rotation model used here is shown to give a more faithful description of the observed velocity field than the linear model based on the Oort constants $A_{G}$ and $B_{G}$. We present evidence that the young clusters $\beta$ Pic, Tuc/HorA, and TWA belong to the Gould Belt structure.
\end{abstract}

Key words: Gould Belt, Galaxy (Milky Way), OB associations, TW Hydrae, $\beta$ Pictoris, Tucana.

\section{INTRODUCTION}

The star-and-gas complexes associated with star formation processes in our Galaxy and in other galaxies have a hierarchical structure (Efremov 1998; Efremov and Elmegreen 1998). Giant star-and-gas (GSG) complexes trace the spiral pattern of the Galaxy (Efremov 1998). The GSG complexes have masses of $\approx 1 \times 10^{6} M_{\odot}$, sizes as large as $1000 \mathrm{pc}$, and lifetimes $\tau<10^{8}$ yr. Less massive structures, such as OB associations, open star clusters, and giant molecular clouds, are part of a GSG complex. The Sun lies within a GSG complex that is known as the Gould Belt (with a radius of $\approx 500 \mathrm{pc}$ and a lifetime of $\tau \approx 60 \times 10^{6} \mathrm{yr}$ ). In turn, the Gould Belt is part of an older $\left(\tau \approx 5 \times 10^{8} \mathrm{yr}\right)$ and more massive $\left(\approx 2 \times 10^{7} M_{\odot}\right)$ structure about $1000 \mathrm{pc}$ in size that is known as the Local (Orion) Arm or the Local system of stars perceived as a gravitationally bound, long-lived system. Its kinematics suggests that it is associated (Olano 2001) with the Sirius star supercluster (Eggen 1984, 1992). Since the mean residual velocity of the Sirius supercluster stars relative to the local standard of rest (LSR) is low, the center of mass of the Local system of stars has made several turns around the Galactic center in a nearly circular orbit. Over its lifetime, the Gould Belt has probably experienced a single impact from a spiral density wave.

An important indicator of the stability of such a system as the Gould Belt is the existence of proper rotation. Such rotation is reliably found from observations of Gould Belt stars (Lindblad 2000; Bobylev 2004). Over its lifetime, the Gould Belt has been affected by the 
shock wave generated by a spiral density wave at least once. An analysis of the motions of the OB associations that fill the interarm space $(r \leq 3 \mathrm{kpc})$ reveals a complex periodic structure of their residual velocities attributable to the influence of spiral density waves (Mel'nik et al. 2001; Zabolotskikh et al. 2002; Mel'nik 2003). One of these features is the prominent structure in the radial residual velocity distribution in Galactocentric distance in the Gould Belt region (see Fig. 3a in Mel'nik et al. 2001) that we associate with the well-known positive K-effect or with the expansion of young stars. In this paper, based on a sample of individual stars that are members of OB associations, we study this effect in detail.

The goal of this work is to study the motion of the Gould Belt using data on stars with reliable age estimates. For this purpose, we use young and nearby OB associations with age estimates obtained by different authors on the basis of currently available observations and methods. The rotation and expansion parameters of the Gould Belt are determined by assuming the existence of a single kinematic center. To this end, we use Bottlinger's formulas in a form that allows the direction of the single kinematic center to be analytically determined simultaneously with the rotation and expansion-contraction parameters.

\section{METHODS OF ANALYSIS \\ Bottlinger's Formulas}

In this paper, we use a rectangular Galactic coordinate system with the axes directed away from the observer toward the Galactic center $\left(l=0^{\circ}, b=0^{\circ}\right.$, the $X$ axis), along the Galactic rotation $\left(l=90^{\circ}, b=0^{\circ}\right.$, the $Y$ axis), and toward the North Galactic Pole ( $b=90^{\circ}$, the $Z$ axis). We derived the basic equations from Bottlinger's standard formulas (Ogorodnikov 1965). By assuming the existence of a single kinematic center of rotation and expansion- contraction $\left(l_{\circ}, R_{\circ}\right)$, we transformed the formulas to

$$
\begin{gathered}
V_{r}=u_{G} \cos b \cos l+v_{G} \cos b \sin l+w_{G} \sin b- \\
-D_{1}\left(R-R_{\circ}\right) \sin \left(l-l_{\circ}\right) \cos b- \\
-D_{2}\left(R-R_{\circ}\right) \cos \left(l-l_{\circ}\right) \cos b- \\
-F_{1}\left(R-R_{\circ}\right)^{2} \sin \left(l-l_{\circ}\right) \cos b- \\
-F_{2}\left(R-R_{\circ}\right)^{2} \cos \left(l-l_{\circ}\right) \cos b+ \\
+k_{\circ} r \cos ^{2} b+k_{\circ}^{\prime} r\left(R-R_{\circ}\right) \cos ^{2} b+ \\
\quad+0.5 k_{\circ}^{\prime \prime} r\left(R-R_{\circ}\right)^{2} \cos ^{2} b, \\
4.74 r \mu_{l} \cos b=-u_{G} \sin l+v_{G} \cos l+ \\
+\omega_{\circ} r \cos b+\omega_{\circ}^{\prime} r\left(R-R_{\circ}\right) \cos b+ \\
\quad+0.5 \omega_{\circ}^{\prime \prime} r \cos b\left(R-R_{\circ}\right)^{2}- \\
-D_{1}\left(R-R_{\circ}\right) \cos \left(l-l_{\circ}\right)+D_{2}\left(R-R_{\circ}\right) \sin \left(l-l_{\circ}\right)- \\
-F_{1}\left(R-R_{\circ}\right)^{2} \cos \left(l-l_{\circ}\right)+F_{2}\left(R-R_{\circ}\right)^{2} \sin \left(l-l_{\circ}\right), \\
4.74 r \mu_{b}=-u_{G} \cos l \sin b-v_{G} \sin l \sin b+w_{G} \cos b+ \\
+D_{1}\left(R-R_{\circ}\right) \sin \left(l-l_{\circ}\right) \sin b+ \\
\quad+D_{2}\left(R-R_{\circ}\right) \cos \left(l-l_{\circ}\right) \sin b+ \\
\quad+F_{1}\left(R-R_{\circ}\right)^{2} \sin \left(l-l_{\circ}\right) \sin b+ \\
+F_{2}\left(R-R_{\circ}\right)^{2} \cos \left(l-l_{\circ}\right) \sin b-
\end{gathered}
$$




$$
\begin{gathered}
-k_{\circ} r \cos b \sin b-k_{\circ}^{\prime} r\left(R-R_{\circ}\right) \cos b \sin b- \\
-0.5 k_{\circ}^{\prime \prime} r\left(R-R_{\circ}\right)^{2} \cos b \sin b .
\end{gathered}
$$

Here, the coefficient 4.74 is the quotient of the number of kilometers in an astronomical unit by the number of seconds in a tropical year, $r=1 / \pi$ is the heliocentric distance of the star, $R_{\circ}$ is the distance from the Sun to the kinematic center, and $R$ is the distance from the star to the kinematic center. Since we corrected the observed motions for the peculiar solar motion with respect to the LSR (Dehnen and Binney 1998), the quantities $u_{G}, v_{G}$, and $w_{G}$ are (in contrast to the standard approach, we reversed the signs) the velocity components of the centroid of the stars under consideration with respect to the LSR; the components of the

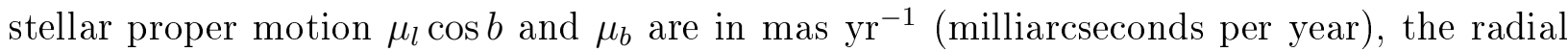
velocity $V_{r}$ is in $\mathrm{km} \mathrm{s}^{-1}$, the parallax $\pi$ is in mas (milliarcseconds), and the distances $R, R_{\circ}$, and $r$ are in kpc. The quantity $\omega_{\circ}$ is the angular velocity of the stellar system at distance $R_{\circ}$; $k_{\circ}$ is the radial expansion (or contraction) rate of the stellar system at distance $R_{\circ} ; \omega_{\circ}^{\prime}, \omega_{\circ}^{\prime \prime}$, and $k_{\circ}^{\prime}, k_{\circ}^{\prime \prime}$ are the corresponding derivatives with respect to the heliocentric distance taken at distance $R_{\circ}$; and $l_{\circ}$ is the direction of the kinematic center. $R$ can be calculated using the expression

$$
R^{2}=(r \cos b)^{2}-2 R_{\circ} r \cos b \cos \left(l-l_{\circ}\right)+R_{\circ}^{2} .
$$

In Eqs. (1), we use the unknowns

$$
\begin{gathered}
D_{1}=D \cos l_{\theta}, \quad D_{2}=-D \sin l_{\theta}, \\
F_{1}=F \cos l_{\theta}, \quad F_{2}=-F \sin l_{\theta},
\end{gathered}
$$

where $D=\sqrt{D_{1}^{2}+D_{2}^{2}}=\omega^{\prime} R_{\circ}$ и $F=\sqrt{F_{1}^{2}+F_{2}^{2}}=0.5 \omega^{\prime \prime} R_{\circ}$. Based on relations (3), we determine the correction $l_{\theta}$ to the assumed value of $l_{\circ}$ twice:

$$
\begin{gathered}
\tan \left(l_{\theta}\right)_{D}=-D_{2} / D_{1}, \\
\tan \left(l_{\theta}\right)_{F}=-F_{2} / F_{1},
\end{gathered}
$$

in this case, the new direction is $l_{\circ}+l_{\theta}$. On the other hand, we may introduce similar quantities, $G=k^{\prime} R_{\circ}$ and $H=0.5 k^{\prime \prime} R_{\circ}$, with the unknowns $G_{1}, G_{2}, H_{1}$, and $H_{2}$ for the expansion to obtain

$$
\begin{gathered}
G_{1}=G \cos l_{R}=-G \sin l_{\theta}, \\
G_{2}=-G \sin l_{R}=G \cos l_{\theta}, \\
H_{1}=H \cos l_{R}=-H \sin l_{\theta}, \\
H_{2}=-H \sin l_{R}=H \cos l_{\theta} .
\end{gathered}
$$

The orthogonality of the expansion and rotation effects $\left(l_{\theta}=l_{R}+90^{\circ}\right)$ is reflected in relations (5); as a result, the unknowns in Eqs. (1) cannot be separated. Therefore, to determine the direction of the kinematic center, it will suffice to use one of the sets of unknowns, for example, $D_{1}, D_{2}, F_{1}$, and $F_{2}$, in Eqs. (1). Based on the described approach, we can independently estimate the distance from the Sun to the kinematic center. This estimate can be obtained from the formulas that follow from relations $(3)-(5)$ :

$$
\begin{aligned}
& D=R_{\circ}\left|\omega_{\circ}^{\prime}-k_{\circ}^{\prime}\right|, \\
& F=0.5 R_{\circ}\left|\omega_{\circ}^{\prime \prime}-k_{\circ}^{\prime \prime}\right| .
\end{aligned}
$$


Equations (1) contain thirteen sought unknowns: $u_{\odot}, v_{\odot}, w_{\odot}, \omega_{\circ}, \omega_{\circ}^{\prime}, \omega_{\circ}^{\prime \prime}, k_{\circ}, k_{\circ}^{\prime}, k_{\circ}^{\prime \prime}, D_{1}$, $D_{2}, F_{1}, F_{2}$, which can be determined by the least-squares method. If the direction of the kinematic center is known, then the equations have the original form

$$
\begin{aligned}
& V_{r}=u_{G} \cos b \cos l+ \\
& +v_{G} \cos b \sin l+w_{G} \sin b-R_{\circ}\left(R-R_{\circ}\right) \times \\
& \times \sin \left(l-l_{\circ}\right) \cos b \omega_{\circ}^{\prime}-0.5 R_{\circ}\left(R-R_{\circ}\right)^{2} \times \\
& \times \sin \left(l-l_{\circ}\right) \cos b \omega_{\circ}^{\prime \prime}+\cos ^{2} b k_{\circ} r+ \\
& +\left(R-R_{\circ}\right)\left(r \cos b-R_{\circ} \cos \left(l-l_{\circ}\right)\right) \times \\
& \times \cos b k_{\circ}^{\prime}+0.5\left(R-R_{\circ}\right)^{2}(r \cos b- \\
& \left.-R_{\circ} \cos \left(l-l_{\circ}\right)\right) \cos b k_{\circ}^{\prime \prime}, \\
& 4.74 r \mu_{l} \cos b=-u_{G} \sin l+ \\
& +v_{G} \cos l-\left(R-R_{\circ}\right)\left(R_{\circ} \cos \left(l-l_{\circ}\right)-\right. \\
& -r \cos b) \omega_{\circ}^{\prime}-0.5\left(R-R_{\circ}\right)^{2}\left(R_{\circ} \cos \left(l-l_{\circ}\right)-\right. \\
& -r \cos b) \omega_{\circ}^{\prime \prime}+r \cos b \omega_{\circ}+R_{\circ}\left(R-R_{\circ}\right) \times \\
& \left.\times \sin \left(l-l_{\circ}\right)\right) k_{\circ}^{\prime}+0.5 R_{\circ}\left(R-R_{\circ}\right)^{2} \times \\
& \left.\times \sin \left(l-l_{\circ}\right)\right) k_{\circ}^{\prime \prime} \text {, } \\
& 4.74 r \mu_{b}=-u_{G} \cos l \sin b- \\
& -v_{G} \sin l \sin b+w_{G} \cos b+R_{\circ}\left(R-R_{\circ}\right) \times \\
& \times \sin \left(l-l_{\circ}\right) \sin b \omega_{\circ}^{\prime}+0.5 R_{\circ}\left(R-R_{\circ}\right)^{2} \times \\
& \times \sin \left(l-l_{\circ}\right) \sin b \omega_{\circ}^{\prime \prime}-\cos b \sin b k_{\circ} r- \\
& -\left(R-R_{\circ}\right)\left(r \cos b-R_{\circ} \cos \left(l-l_{\circ}\right)\right) \times \\
& \times \sin b k_{\circ}^{\prime}-0.5\left(R-R_{\circ}\right)^{2}(r \cos b- \\
& \left.-R_{\circ} \cos \left(l-l_{\circ}\right)\right) \sin b k_{\circ}^{\prime \prime} .
\end{aligned}
$$

These equations contain nine sought unknowns: $u_{\odot}, v_{\odot}, w_{\odot}, \omega_{\circ}, \omega_{\circ}^{\prime}, \omega_{\circ}^{\prime \prime}, k_{\circ}, k_{\circ}^{\prime}, k_{\circ}^{\prime \prime}$. A peculiarity of this method is that it requires the existence of derivatives only with respect to the distance. A sample of stars uniformly distributed even in one Galactic quadrant can satisfy this requirement.

\section{The Statistical Method}

We use the standard statistical method (Trümpler and Weaver 1953; Parenago 1954; Ogorodnikov 1965) that consists in determining and analyzing the symmetric moment tensor or the stellar residual velocity dispersion tensor. When both the radial velocities and proper motions of stars are used to determine the six unknown components of the dispersion tensor, we have six equations that can be written for each star. The semiaxes of the residual velocity (Schwarzschild) ellipsoid that we denote by $\sigma_{1,2,3}$ can be determined by analyzing the eigenvalues of the dispersion tensor. We denote the directions of the principal axes of this ellipsoid by $l_{1,2,3}$ and $b_{1,2,3}$. A peculiarity of the approach used here is that the stellar velocities corrected for the peculiar solar motion with respect to the LSR and for the general Galactic rotation are used as the residual velocities. 


\section{The Ogorodnikov-Milne Model}

In the linear Ogorodnikov-Milne model, we use the same notation that was introduced by Clube $(1972,1973)$ and used by du Mont $(1977,1978)$. We modify the linear model developed to describe the general Galactic rotation to describe the peculiarities of the velocity field of nearby stars. To within terms of the first order of smallness $r / R_{\circ} \ll 1$, the observed velocity $\mathbf{V}(r)$ of a star with a heliocentric radius vector $r$ is described by the vector equation

$$
\mathbf{V}(r)-\mathbf{V}_{G R}-\mathbf{V}_{\odot L S R}=\mathbf{V}_{G}+M \mathbf{r}+\mathbf{V}^{\prime}
$$

Here, $\mathbf{V}_{G R}$ is the systematic velocity of the star attributable to the general Galactic rotation, $\mathbf{V}_{L S R}$ is the peculiar solar motion with respect to the $\mathrm{LSR}, \mathbf{V}_{G}\left(u_{G}, v_{G}, w_{G}\right)$ is the velocity of the centroid of the stars under consideration relative to the LSR, $\mathbf{V}^{\prime}$ is the residual stellar velocity (the residual stellar velocities are assumed to be distributed randomly), and $M$ is the displacement matrix that describes systematic motions similar to the proper rotation and expansion-contraction effects. The components of the matrix $M$ are the partial derivatives of $\mathbf{u}\left(u_{1}, u_{2}, u_{3}\right)$ with respect to $\mathbf{r}\left(r_{1}, r_{2}, r_{3}\right)$ :

$$
M_{p q}=\left(\frac{\partial u_{p}}{\partial r_{q}}\right)_{\circ}, \quad(p, q=1,2,3) .
$$

The matrix $M$ can be separated into symmetric, $M^{+}$, and antisymmetric, $M^{-}$, parts. Following Ogorodnikov (1965), we call them the local deformation and local rotation tensors, respectively:

$$
\begin{gathered}
M_{p q}^{+}=\frac{1}{2}\left(\frac{\partial u_{p}}{\partial r_{q}}+\frac{\partial u_{q}}{\partial r_{p}}\right)_{\circ}, \\
M_{p q}^{-}=\frac{1}{2}\left(\frac{\partial u_{p}}{\partial r_{q}}-\frac{\partial u_{q}}{\partial r_{p}}\right)_{\circ}, \\
(p, q=1,2,3) .
\end{gathered}
$$

The basic equations are

$$
\begin{gathered}
V_{r}=u_{G} \cos b \cos l+v_{G} \cos b \sin l+w_{G} \sin b+ \\
+r\left(\cos ^{2} b \cos ^{2} l M_{11}+\cos ^{2} b \cos l \sin l M_{12}+\right. \\
+\cos b \sin b \cos l M_{13}+\cos ^{2} b \sin l \cos l M_{21}+ \\
+\cos ^{2} b \sin ^{2} l M_{22}+\cos b \sin b \sin l M_{23}+ \\
+\sin b \cos b \cos l M_{31}+\cos b \sin b \sin l M_{32}+ \\
\left.+\sin ^{2} b M_{33}\right) \\
+4.74 r \mu_{l} \cos b=-u_{G} \sin l+v_{G} \cos l+ \\
+r\left(-\cos b \cos l \sin l M_{11}-\cos b \sin ^{2} l M_{12}-\right. \\
-\sin b \sin l M_{13}+\cos b \cos ^{2} l M_{21}+ \\
+\cos b \sin l \cos l M_{22}+ \\
\left.+\sin b \cos l M_{23}\right)
\end{gathered}
$$




$$
\begin{gathered}
4.74 r \mu_{b}=-u_{G} \cos l \sin b-v_{G} \sin l \sin b+w_{G} \cos b+ \\
+r\left(-\sin b \cos b \cos ^{2} l M_{11}-\right. \\
-\sin b \cos b \sin l \cos l M_{12}- \\
-\sin ^{2} b \cos l M_{13}-\sin b \cos b \sin l \cos l M_{21}- \\
-\sin b \cos b \sin ^{2} l M_{22}-\sin ^{2} b \sin l M_{23}+ \\
+\cos ^{2} b \cos l M_{31}+\cos ^{2} b \sin l M_{32}+ \\
\left.+\sin b \cos b M_{33}\right)
\end{gathered}
$$

where the stellar velocity components corrected for the general Galactic rotation and for the peculiar solar motion with respect to the LSR appear on the left-hand sides. Equations (11) contain twelve sought unknowns: the three velocity components $V_{G}\left(u_{G}, v_{G}, w_{G}\right)$ and the nine components $M_{p g}$ that can be determined by the least-squares method. The deformation and rotation tensor components can be calculated using the values of Mpq derived from relations (10). This method requires the existence of all derivatives with respect to the coordinates. A sample of stars that densely fill each coordinate axis is needed to meet this requirement.

\section{OBSERVATIONAL MATERIAL}

\section{Data on Stars}

We compiled our working list of stars located no farther than $\approx 650$ pc from the Sun using the following sources: we took the Hipparcos (ESA 1997) star numbers for the Col 121, Per OB2, Vel OB2, Tr 10, LCC, UCL, US, Cep OB2, Lac OB1, Cep OB6, $\beta$ Per (Per OB3), and Cas Tau associations from de Zeeuw et al. (1999); for the Ori OB1 a-d associations from Brown et al. (1994); for the IC 2391, IC 2602, NGC 2232, NGC 2451, NGC 2516, NGC 2547, and Pleiades clusters from the list by Robichon et al. (1999); the list of members of the TW Hydrae (TWA), $\beta$ Pic, and Tucana/HorA associations from Song et al. (2003); and for the a Car cluster from Platais et al. (1998). The data on 24 stars having X-ray emissions and located no farther than 50 pc from the Sun were taken from Makarov (2003), where they are denoted as an XY sample. The star HIP 30030 in Makarov's list is a member of the TWA cluster, belongs to the Tucana/HorA group, and is designated TWA42 (Song et al. 2003). The data on 34 stars located no farther than 100 pc from the Sun were taken from Wichmann et al. (2000, 2003). Four stars were not included in other lists; we placed them in group 2. Table 1 gives the ages of the selected clusters. We took the equatorial coordinates, parallaxes, and proper motions from the Hipparcos catalog and the radial velocities from the catalog by Barbier-Brossat and Figon (2000). We use only single stars (the astrometric orbital binaries marked by the symbol $\mathrm{O}$ were rejected) for which the parallaxes, radial velocities, and proper motions are available. Based on the stellar ages (Table 1), we divided the stars into three groups: (1) the youngest stars with ages $<10$ Myr (group 1); (2) the stars with middle ages of $10-60 \mathrm{Myr}$ (group 2); and (3) the old stars with ages of $60-125$ Myr (group 3).

\section{Allowance for the Galactic Rotation}

We took into account the general Galactic rotation using the parameters found previously (Bobylev 2004): $\omega_{\circ}=-28.0 \pm 0.6 \mathrm{~km} \mathrm{~s}^{-1} \mathrm{kpc}^{-1}, \omega_{\circ}^{\prime}=+4.17 \pm 0.14 \mathrm{~km} \mathrm{~s}^{-1} \mathrm{kpc}^{-2}$, and $\omega_{\circ}^{\prime \prime}=-0.81 \pm 0.12 \mathrm{~km} \mathrm{~s}^{-1} \mathrm{kpc}^{-3}$. The Galactocentric distance of the Sun was assumed to be $R_{\circ}=7.1 \mathrm{kpc}$, which corresponds to the short distance scale (Dambis et al. 2001). The 
radial velocities and proper motions of the stars were corrected for peculiar solar motion with respect to the LSR using the values obtained by Dehnen and Binney $(1998):\left(u_{\odot}, v_{\odot}, w_{\odot}\right)=$ $(10.0,5.3,7.2) \mathrm{km} \mathrm{s}^{-1}$.

\section{RESULTS}

A preliminary analysis of the stellar space velocities showed the necessity of setting a limit on the total stellar velocity, $\sqrt{U^{2}+V^{2}+W^{2}}<30 \mathrm{~km} \mathrm{~s}^{-1}$, which we use below. The residual velocities $U, V$, and $W$ were calculated from standard formulas (Kulikovskii 1985). We used stars with parallaxes $\pi>1.5$ mas $(r<667 \mathrm{pc})$. The number of stars used is given in the last column of Table 1. Thus, each of groups 1, 2, and 3 always contains a fixed number of stars, 114, 342, and 150, respectively. For our sample of stars, the mean error in the parallax is $15-17 \%$. The mean errors in the proper motion components of the stars $4.74 r \mu_{l} \cos b$ and $4.74 r \mu_{b}$, including the errors in the parallaxes, are $\approx 1 \mathrm{~km} \mathrm{~s}^{-1}$. For $\approx 40 \%$ of the stars, there are no data on the errors in the radial velocities (Barbier-Brossat and Figon 2000); for stars with available information about the errors, the mean error is $\approx 3.5$ $\mathrm{km} \mathrm{s}^{-1}$.

The group 1 stars are distributed exclusively in Galactic quadrant III. The group 2 stars fill the solar neighborhood within $r \approx 650$ pc of the Sun most uniformly. The group 3 stars densely fill a compact zone within $r \approx 50 \mathrm{pc}$ of the Sun in Galactic quadrant II. We also formed a combined group of young $(<60 \mathrm{Myr})$ stars composed of the group 1 and group 2 stars. For the latter combined group, the application of all kinematic models proved to be possible.

As the first approximation for the direction of the kinematic center, we take $l_{\circ}=160^{\circ}$ and $R_{\circ}=150$ pc (Bobylev 2004). Solving the system of equations (1) for the combined group of young stars (group 1+group 2) yielded the following kinematic parameters of the linear motion of the stars: $\left(u_{G}, v_{G}, w_{G}\right)=(0.0 \pm 0.7,-12.3 \pm 0.7,1.3 \pm 0.3) \mathrm{km} \mathrm{s}^{-1}$. Consequently, the stars under consideration move with respect to the LSR at a velocity of $V_{G}=12.3 \pm 0.7$ $\mathrm{km} \mathrm{s}^{-1}$ in the direction $L_{G}=270 \pm 3^{\circ}$ and $B_{G}=6 \pm 1^{\circ}$. Further,

$$
\begin{gathered}
\omega_{\circ}=-32.4 \pm 3.8 \mathrm{~km} \mathrm{~s}^{-1} \mathrm{kpc}^{-1}, \\
\omega_{\circ}^{\prime}=+93.2 \pm 28.3 \mathrm{~km} \mathrm{~s}^{-1} \mathrm{kpc}^{-2}, \\
\omega_{\circ}^{\prime \prime}=-170.5 \pm 107.8 \mathrm{~km} \mathrm{~s}^{-1} \mathrm{kpc}^{-3}, \\
k_{\circ}=+27.9 \pm 3.8 \mathrm{~km} \mathrm{~s}^{-1} \mathrm{kpc}^{-1}, \\
k_{\circ}^{\prime}=-122.3 \pm 28.3 \mathrm{~km} \mathrm{~s}^{-1} \mathrm{kpc}^{-2}, \\
k_{\circ}^{\prime \prime}=+233.7 \pm 107.8 \mathrm{~km} \mathrm{~s}^{-1} \mathrm{kpc}^{-3}, \\
l_{\circ}=128^{\circ}, \\
\left(l_{\theta}\right)_{D}=2^{\circ}, \\
\left(l_{\theta}\right)_{F}=181^{\circ}, \\
\left(R_{\circ}\right)_{D}=180 \mathrm{pc}, \\
\left(R_{\circ}\right)_{F}=410 \mathrm{pc} .
\end{gathered}
$$

The results of the solution of (12) are presented in Fig. 1, where line 1 indicates the derived expansion curve with the estimated parameters $k_{\circ}, k_{\circ}^{\prime}$, and $k_{\circ}^{\prime \prime}$; line 2 indicates the rotation curve with the estimated parameters $\omega_{\circ}, \omega_{\circ}^{\prime}$, and $\omega_{\circ}^{\prime \prime}$. As we see from the solution of (12), the value of $R_{\circ}=180 \mathrm{pc}$ calculated using the first derivatives is in satisfactory 
agreement with the $R_{\circ}=150 \mathrm{pc}$ found previously (Bobylev 2004). The expansion curve constructed by solving (12) has a velocity maximum of $V=4.4 \mathrm{~km} \mathrm{~s}^{-1}$ at $R=200 \mathrm{pc}$ from the center. Based on these data, we determined the time elapsed from the onset of expansion, $\tau=44 \mathrm{Myr}$, and it is in good agreement with the ages of the stars under consideration.

Applying the Ogorodnikov-Milne model (Eqs. (11)) to the combined group of young stars (group $1+$ group 2), we found the following components of the tensors $M, M^{+}$, and $M^{-}(\mathrm{km}$ $\left.\mathrm{s}^{-1} \mathrm{kpc}^{-1}\right)$ :

$$
\begin{gathered}
M=\left(\begin{array}{ccc}
13.0_{(3.3)} & 8.1_{(1.4)} & -4.4_{(8.6)} \\
-21.5_{(3.3)} & 2.9_{(1.4)} & -7.0_{(8.6)} \\
4.6_{(3.3)} & 4.3_{(1.4)} & -18.8_{(8.6)}
\end{array}\right), \\
M^{+}=\left(\begin{array}{ccc}
13.0_{(3.3)} & -6.7_{(1.8)} & 0.1_{(4.6)} \\
-6.7_{(1.8)} & 2.9_{(1.4)} & -1.4_{(4.3)} \\
0.1_{(4.6)} & -1.4_{(4.3)} & -18.8_{(8.6)}
\end{array}\right), \\
M^{-}=\left(\begin{array}{ccc}
0 & 14.8_{(1.8)} & -7_{(5.0)} \\
-14.8_{(1.8)} & 0 & -4.5_{(4.6)} \\
4.5_{(4.6)} & 5.7_{(4.3)} & 0
\end{array}\right) .
\end{gathered}
$$

The Oort parameters are: $A_{G}=M_{21}^{+}=-6.7 \pm 1.8 \mathrm{~km} \mathrm{~s}^{-1} \mathrm{kpc}^{-1}, B_{G}=M_{21}^{-}=-14.8 \pm 1.8 \mathrm{~km}$ $\mathrm{s}^{-1} \mathrm{kpc}^{-1}, C_{G}=0.5\left(M_{11}^{+}-M_{22}^{+}\right)=5.1 \pm 1.8 \mathrm{~km} \mathrm{~s}^{-1} \mathrm{kpc}^{-1}, K_{G}=0.5\left(M_{11}^{+}+M_{22}^{+}\right)=8.0 \pm 1.8$ $\mathrm{km} \mathrm{s}^{-1} \mathrm{kpc}^{-1}$. In Eqs. (11), the $X$ axis is directed toward the Galactic center $\left(l_{\circ}=0^{\circ}\right)$. The deformation tensor in the principal axes $M_{G}^{+}$is $\left(\mathrm{km} \mathrm{s}^{-1} \mathrm{kpc}^{-1}\right)$ :

$$
M_{G}^{+}=\left(\begin{array}{ccc}
16.4 & 0 & 0 \\
0 & -0.4 & 0 \\
0 & 0 & -18.9
\end{array}\right)
$$

the directions of the principal axes are:

$$
\begin{gathered}
L_{1}=153.5 \pm 0.2^{\circ}, \quad B_{1}=-1.1 \pm 0.0^{\circ}, \\
L_{2}=243 \pm 10^{\circ}, \quad B_{2}=4 \pm 8^{\circ}, \\
L_{3}=81 \pm 10^{\circ}, \quad B_{3}=86 \pm 11^{\circ} .
\end{gathered}
$$

The vertex deviation in the $X Y$ plane that we determined from the formula $\tan 2 l_{X Y}=$ $-C / A$ is $l_{X Y}=19 \pm 6^{\circ}$ and indicates one of the directions of the rotation center; the other direction is $l=109^{\circ}$ and is close to the direction of the kinematic center $l_{\circ}=128^{\circ}$ that we found using Eqs. (1).

An analysis of the deformation tensor (19), (20) leads us to the important conclusion (which cannot be drawn from model (1)) that expansion takes place exclusively in the Galactic $X Y$ plane.

The results of applying the statistical method are given in Table 2. As we see from this table, the direction of the rotation center for the combined group of stars (Group 1+Group 2) is determined accurately, $l_{1}=132.7 \pm 0.4^{\circ}$. The first axis of the residual velocity ellipsoid (the vertex deviation) changes its direction with the age of the stars under consideration. This is consistent with the dynamic model of Olano (2001). As Olano showed, the direction of the vertex rotation depends on the critical stellar density in the solar neighborhood $\rho / \rho^{*}$, where $\rho$ is given in $M_{\odot} \mathrm{pc}^{-3}$. Following Olano's estimates $\left(\rho / \rho^{*}=1.5\right)$, we assume that the change occurs counterclockwise in the coordinate system under consideration. Thus, the 
vertex deviation changes from $l_{1}=36^{\circ}$ for the sample of old stars (Group 3 ) to $l_{1}=131^{\circ}$ for the sample of middle-aged stars (Group 2) and to $l_{1}=172^{\circ}$ for the sample of the youngest stars (Group 1). Associating this direction with the direction of the kinematic center, we can see that the center does not remain constant. Therefore, its independent determination is required for each sample of stars. There is good agreement of the orientation of the residual velocity tensor for the Group 1 and Group 2 stars that we found (Table 2) with the geometric characteristics of the Gould Belt (Torra et al. 2000). For example, for the group-2 stars, the directions $l_{2}=224 \pm 18^{\circ}$ and $b_{2}=-22 \pm 5^{\circ}$ almost match the characteristics of the Gould Belt $\Omega_{G}=275-295^{\circ}$ and $i_{G}=16-20^{\circ}$ (here, $\Omega_{G}$ is the direction of the line of nodes of the Gould Belt and $i_{G}$ is the inclination of the disk to the Galactic plane) found by Torra et al. (2000).

For the combined group of stars (Group 1+Group 2), we obtained the solutions of Eqs. (7) in which we used $l_{\circ}=128^{\circ}$ and $R_{\circ}=150$ pc. Equations $(7)$ were solved for two cases, with nine and seven unknowns in the first and second cases, by assuming that the second derivatives $\omega_{\circ}^{\prime \prime}$ and $k_{\circ}^{\prime \prime}$ are equal to zero. The results are presented in Table 3 and Fig. 2. In Fig. 2, curves 1 and 2 were constructed using the data in the upper part of Table. 3 ; curves 5 and 6 were constructed using the data in the lower part of Table 3 (seven unknowns). Curve 4 in Fig. 2 represents the results of applying the Oort-Lindblad model, which for $l_{\circ}=128^{\circ}$ are the following: $A_{G}=6.5 \pm 1.8 \mathrm{~km} \mathrm{~s}^{-1} \mathrm{kpc}^{-1}$ and $B_{G}=-14.8 \pm 1.8 \mathrm{~km} \mathrm{~s}^{-1} \mathrm{kpc}^{-1}$ (these values were obtained from the equations of the Ogorodnikov- Milne model, but the curve is essentially the Oort-Lindblad approximation). The time elapsed from the onset of expansion for curves 2 and 6 is about $\tau=120 \mathrm{Myr}$, which significantly exceeds the estimate obtained by solving (12).

The results of solving Eqs. (7) for group 3 are given in the lower part of Table 3. The parameters derived for group 3 differ significantly from those of young stars: the rotation and the expansion have opposite signs. We associate the kinematic peculiarities of the Local system of stars that were found by Tsvetkov (1995a, 1995b, 1997) by studying type-A and -F stars with these stars. It is the influence of the Group 3 stars that led to the paradox that younger OB stars showed a slower rotation than older stars (Bobylev 2004).

Figures 3 and 4 show the residual velocities $U, V$, and $W$ of middle-aged Gould Belt stars in projection onto the Galactic $X Y$ and $X Z$ planes. These velocities are residual in every sense, because they were corrected from both the peculiar solar motion and the total velocity $V_{G}$ that we found. Thus, we relate the velocities to a coordinate system whose center is the center of mass of the Gould Belt that moves with respect to the LSR.

Figure 5 shows the residual velocities of stars in the young $\beta$ Pic and Tuc/HorA clusters together with TWA cluster stars.

\section{DISCUSSION}

The kinematic parameters that we derived using the linear Ogorodnikov-Milne model are in excellent agreement with the kinematic model of the Gould Belt suggested by Lindblad (2000), which is based on an analysis of the results by Comeron (1999) and Torra et al. (1997) for the age interval of Gould Belt stars 20 - 40 Myr. Lindblad's model assumes the proper rotation of the Gold Belt stars with an angular velocity of $\omega_{G}=B-A_{\omega}=-24$ $\mathrm{km} \mathrm{s}^{-1} \mathrm{kpc}^{-1}\left(A_{\omega}=6.4 \mathrm{~km} \mathrm{~s}^{-1} \mathrm{kpc}^{-1}\right.$ and $\left.B=-17.4 \mathrm{~km} \mathrm{~s}^{-1} \mathrm{kpc}^{-1}\right)$ and the expansion with $K=11.3 \mathrm{~km} \mathrm{~s}^{-1} \mathrm{kpc}^{-1}$ in the direction of the center $l_{\circ}=127^{\circ}$. An analysis of the curves in Fig. 2 indicates that the linear model faithfully describes the observed velocity 
field of the stars under consideration no farther than 200-300 pc from the kinematic center. Our rotation parameters for the Gould Belt are consistent with the model by Olano (2001) and with the evolution model of the youngest moving clusters by Asiain et al. (1999). The question of whether the $\beta$ Pic, Tuc/HorA, and TWA clusters belong to the Gould Belt structure is currently being debated. Makarov and Fabricius (2001) directly associate these clusters with the Gould Belt; Song et al. (2003) cast doubt on this association. In our opinion, a comparison of the stellar residual velocities for all of the clusters ( $\beta$ Pic, Tuc/HorA, and TWA) in Fig. 5 with our rotation and expansion curves suggests that the cluster stars belong to the Gould Belt structure. The last row in Table 2 gives our calculated parameters of the residual velocity ellipsoid for all 33 stars of the $\beta$ Pic, Tuc/HorA, and TWA clusters. An analysis of $l_{1}, b_{1}, l_{2}, b_{2}, l_{3}, b_{3}$ and a comparison of Figs. 4 and 5 indicate that these stars belong to the disk of the Gould Belt. Ortega et al. (2002) performed model calculations of the orbits of $\beta$ Pic cluster members in the Galactic field of attraction for an interval of 11.5 Myr and used them to determine the birthplace of the cluster. As follows from an analysis of our rotation curve for the Gould Belt (line 1 in Fig. 2), the Gould Belt has a substantial mass. Therefore, in our view, to determine the birthplaces of the $\beta$ Pic, Tuc/HorA, and TWA clusters, the gravitational potential of the Gould Belt $\left(\approx 2 \times 10^{6} M_{\odot}\right)$ or the entire Local system of stars $\left(\approx 2 \times 10^{7} M_{\odot}\right)$ must be taken into account.

\section{CONCLUSIONS}

We have analyzed the residual velocity field of nearby and young Gould Belt stars by assuming the existence of a single kinematic center (the center of proper rotation and expansion). The direction of the kinematic center of the Gould Belt was found to be: $l_{\circ}=128^{\circ}$ and $R_{\circ}=150 \mathrm{pc}$.

Using the Ogorodnikov-Milne model, we showed that the vectors of the radial component of the residual velocity field (a positive $K$-effect) lie in the Galactic $X Y$ plane unrelated to the plane of symmetry of the Gould Belt disk. This led us to conclude that the effect in question results from the impact of a spiral density wave propagating precisely through the Galactic disk on the cloud of gas out of which the stars under consideration were subsequently formed. In contrast, the effect of proper rotation is closely related to the plane of symmetry of the Gould Belt disk.

We have considered an approach that, in our view, allows the observed residual velocity field of the Gould Belt stars to be described more faithfully than does the linear (Oort-Lindblad) model. The linear velocities that we found for a sample of stars younger than 60 Myr reach their maximum at a distance of $\approx 300 \mathrm{pc}$ from the kinematic center and are $-6 \mathrm{~km} \mathrm{~s}^{-1}$ for the rotation and $+4 \mathrm{~km} \mathrm{~s}^{-1}$ for the expansion. Our constructed proper rotation curve for the sample of young stars in the Gould Belt suggests that its mass is substantial. This conclusion is in agreement with the spatial distribution of faint stars with X-ray emission (Guillout et al. 1998). We argue that the young $\beta$ Pic, Tuc/HorA, and TWA clusters belong to the Gould Belt structure.

\section{ACKNOWLEDGMENTS}

I am grateful to A. T. Bajkova for her help and useful discussions. This work was supported by the Russian Foundation for Basic Research (project no. 02-02-16570).

\section{REFERENCES}


1. R. Asiain, F. Figueras, and J. Torra, Astron. Astrophys. 350, 434 (1999).

2. M. Barbier-Brossat and P. Figon, Astron. Astrophys., Suppl. Ser. 142, 217 (2000).

3. V. V. Bobylev, Pis'ma Astron. Zh. 30, 185 (2004) [Astron. Lett. 30, 159 (2004)].

4. A. G. A. Brown, E. J. de Geus, and P. T. de Zeeuw, Astron. Astrophys. 289, 101 (1994).

5. F. Carrier, G. Burki, and C. Richard, Astron. Astrophys. 341, 469 (1999).

6. S. V. M. Clube, Mon. Not. R. Astron. Soc. 159, 289 (1972).

7. S. V. M. Clube, Mon. Not. R. Astron. Soc. 161, 445 (1973).

8. F. Comerón, Astron. Astrophys. 351, 506 (1999).

9. A. K. Dambis, A. M. Mel'nik, and A. S. Rastorguev, Pis'ma Astron. Zh. 27, 68 (2001) [Astron. Lett. 27, 58 (2001)].

10. W. Dehnen and J. J. Binney, Mon. Not. R. Astron. Soc. 298, 387 (1998).

11. P. T. de Zeeuw, R. Hoogerwerf, J. H. J. de Bruijne, et al., Astron. J. 117, 354 (1999).

12. B. du Mont, Astron. Astrophys. 61, 127 (1977).

13. B. du Mont, Astron. Astrophys. 66, 441 (1978).

14. Y. N. Efremov, Astron. Astrophys. Trans. 15, 3 (1998).

15. Y. N. Efremov and B. G. Elmegreen, Mon. Not. R. Astron. Soc. 299, 588 (1998).

16. O. J. Eggen, Astron. J. 89, 1350 (1984).

17. O. J. Eggen, Astron. J. 104, 1493 (1992).

18. A. Giménez and J. V. Clauzen, Astron. Astrophys. 291, 795 (1994).

19. P. Guillout, M. F. Sterzik, J. H.M.M. Schmitt, et al., Astron. Astrophys. 337, 113 (1998).

20. R. D. Jeffries and A. J. Tolley, Mon. Not. R. Astron. Soc. 300, 331 (1998).

21. P. G. Kulikovskii, Stellar Astronomy (Nauka, Moscow, 1985) [in Russian].

22. K. L. Luhman, Astrophys. J. 560, 287 (2001).

23. P. O. Lindblad, Astron. Astrophys. 363, 154 (2000).

24. V. V. Makarov, Astron. J. 126, 1996 (2003).

25. V. V. Makarov and C. Fabricius, Astron. Astrophys. 368, 866 (2001).

26. E. E. Mamajek, M. Meyer, and J. Liebert, Astron. J. 124, 1670 (2002).

27. A. M. Mel'nik, Pis'ma Astron. Zh. 29, 349 (2003) [Astron. Lett. 29, 304 (2003)].

28. A. M. Mel'nik, A. K. Dambis, and A. S. Rastorguev, Pis'ma Astron. Zh. 27, 611 (2001) [Astron. Lett. 27, 521 (2001)].

29. D. Montes, J. Loṕez-Santiago,M. C. Gálvez, et al., Mon. Not. R. Astron. Soc. 328, 45 (2001).

30. K. F. Ogorodnikov, Dynamics of Stellar Systems (Fizmatgiz, Moscow, 1965) [in Russian].

31. C. A. Olano, Astron. J. 121, 295 (2001).

32. V. G. Ortega, de la Reza, E. Jilinski, et al., Astrophys. J. Lett. 575, L75 (2002).

33. P. P. Parenago, A Course on Stellar Astronomy (Gosizdat, Moscow, 1954) [in Russian].

34. I. Platais, V. Kozhurina-Platais, and F. van Leeuwen, Astron. J. 116, 2423 (1998).

35. S. Randich, N. Aharpour, R. Pallavicini, et al., Astron. Astrophys. 323, 86 (1997).

36. N. Robichon, F. A. Arenou, J.-C. Mermilliod, et al., Astron. Astrophys. 345, 471 (1999).

37. M. J. Sartori, J. R. D. Ler pine, andW. S. Dias, Astron. Astrophys. 404, 913 (2003).

38. D. R. Soderblom, B. F. Jones, S. Balachandran, et al., Astron. J. 106, 1059 (1993).

39. I. Song, B. Zuckermann, and M. S. Bessel, Astrophys. J. 599, 342 (2003). 
40. J. R. Stauffer, L. W. Hartmann, C. F. Prosser, et al., Astrophys. J. 479, 776 (1997).

41. The Hipparcos and Tycho Catalogues, ESA SP-1200 (1997).

42. J. Torra, D. Fern ar ndez, and F. Figueras, Astron. Astrophys. 359, 82 (2000).

43. J. Torra, A. E. Gómez, F. Figueras, et al., HIPPARCOS Venice'97, Ed. by B. Battrick (ESA Publ. Div., Noordwijk, 1997), p. 513.

44. R. J. Trümpler and H. F.Weaver, Statistical Astronomy (Univ. of California Press, Berkely, 1953).

45. A. S. Tsvetkov, Astron. Astrophys. Trans. 8, 145 (1995).

46. A. S. Tsvetkov, Astron. Astrophys. Trans. 9, 1 (1995).

47. A. Tsvetkov, JOURNEES-97, Ed. by J. Vondrák and N. Capitaine (Observatoire de Paris, Paris, 1997), p. 171.

48. R. Wichmann, J. H. M. M. Schmitt, and S. Hubrig, Astron. Astrophys. 399, 983 (2003).

49. R. Wichmann, G. Torres, C. H. F. Melo, et al., Astron. Astrophys. 359, 181 (2000).

50. M. V. Zabolotskikh, A. S. Rastorguev, and A. K. Dambis, Pis'ma Astron. Zh. 28, 516 (2002) [Astron. Lett. 28, 454 (2002)]. 
Table 1. Data on nearby clusters and OB associations

\begin{tabular}{|c|c|c|c|}
\hline Cluster/association name & Age, $\mathrm{Myr}$ & Reference & $N_{*}$ \\
\hline \multicolumn{4}{|l|}{ Group 1} \\
\hline Ori OBla & $11.4 \pm 1.9$ & Brown et al. (1994) & 29 \\
\hline Ori OB1b & $1.7 \pm 1.1$ & Brown et al. (1994) & 20 \\
\hline Ori OBlc & $4.6 \pm 2$ & Brown et al. (1994) & 25 \\
\hline Ori OBld & $<1$ & Brown et al. (1994) & 3 \\
\hline $\mathrm{Col} 121$ & 5 & de Zeeuw et al. (1999) & 20 \\
\hline TW Hydrae (TWA) & $\leq 10$ & Song et al. (2003)(f) & 17 \\
\hline \multicolumn{4}{|l|}{ Group 2} \\
\hline Per OB2 & $2-15$ & Jiménez and Clausen (1994) & 9 \\
\hline NGC 2232 & $30-60$ & Torra et al. (2000) & 4 \\
\hline NGC 2451 & 50 & Carrier et al. (1999) (c) & 5 \\
\hline NGC 2516 & $30-60$ & Torra et al. (2000) & 6 \\
\hline NGC 2547 & $30-40$ & Jefirries and Tolley (1998) & 5 \\
\hline Vel OB2 & 20 & de Zeeuw et al. (1999) & 29 \\
\hline IC 2391 & $35-55$ & Montes et al. (2001) (d) & 10 \\
\hline $\operatorname{Tr} 10$ & $15-30$ & de Zeeuw et al. (1999)(c) & 6 \\
\hline a Car & $<60$ & Platais et al. (1998)(c) & 4 \\
\hline IC 2602 & 30 & Luhman (2001) (c, d, g) & 8 \\
\hline LCC & $16-20$ & Sartori et al. (2003) (a) & 46 \\
\hline UCL & $16-20$ & Sartori et al. (2003) (b) & 67 \\
\hline US & $8-10$ & Sartori et al. (2003) & 44 \\
\hline Cep OB2 & $5-10$ & de Zeeuw et al. (1999) & 16 \\
\hline Lac OBI & $15-25$ & de Zeeuw et al. (1999) & 29 \\
\hline$\beta$ Pic & $<30$ & Song et al. (2003) & 8 \\
\hline Tucana/HorA & $<30$ & Song et al. $(2003)(\mathrm{h})$ & 8 \\
\hline Макаров (XY) & $<60$ & Makarov (2003) & 24 \\
\hline Wichmann-1 & $<14$ & Wichmann et al. (2000) & 2 \\
\hline Wichmann-2 (ZAMS) & $\ll 90$ & Wichmann et al. (2003) & 2 \\
\hline \multicolumn{4}{|l|}{ Group 3} \\
\hline Cep OB6 & $\approx 50$ & de Zeeuw et al. (1999) (e) & 10 \\
\hline$\alpha \operatorname{Per}(\operatorname{Per} \mathrm{OB} 3)$ & $\approx 50$ & de Zeeuw et al. (1999) & 46 \\
\hline Cas Tau & $\approx 50$ & de Zeeuw et al. (1999)(e) & 56 \\
\hline Pleiades & $70-125$ & Soderblom et al. (1993) & 14 \\
\hline Wichmann (PL) & $\approx 100$ & Wichmann et al. (2003) & 30 \\
\hline
\end{tabular}

Note: (a) 17-20 Myr (Mamajek et al. 2002); (b) 15-22 Myr (Mamajek et al. 2002); (c) 30-60 Myr (Torra et al. 2000); (d) 30 Myr (Staufier et al. 1997); (e) 50-60 Myr (Asiain et al. 1999); (f) 10 Myr (Luhman 2001);(g) $30 \mathrm{Myr}$ (Randich et al. 1997);(h)30+20 Myr (Luhman 2001). 
Table 2. Principal semiaxes of the residual velocity ellipsoid $\sigma_{1,2,3}$ and their directions $l_{1,2,3}, b_{1,2,3}$

\begin{tabular}{|c|c|c|c|c|}
\hline Group & Age, $\mathrm{Myr}$ & $\sigma_{1}, \sigma_{2}, \sigma_{3}, \mathrm{~km} \mathrm{~s}^{-1}$ & $l_{1}, l_{2}, l_{3}$ & $b_{1}, b_{2}, b_{3}$ \\
\hline \multirow[t]{3}{*}{$1+2$} & $<60$ & $9.1 \pm 1.9$ & $132.7^{\circ} \pm 0.4^{\circ}$ & $-6.0^{\circ} \pm 0.0^{\circ}$ \\
\hline & & $6.8 \pm 1.9$ & $225^{\circ} \pm 27^{\circ}$ & $-19^{\circ} \pm 4^{\circ}$ \\
\hline & & $2.8 \pm 1.9$ & $206^{\circ} \pm 27^{\circ}$ & $70^{\circ} \pm 3^{\circ}$ \\
\hline \multirow[t]{3}{*}{1} & $<10$ & $7.6 \pm 2.3$ & $172^{\circ} \pm 1^{\circ}$ & $-10^{\circ} \pm 2^{\circ}$ \\
\hline & & $7.2 \pm 2.3$ & $263^{\circ} \pm 42^{\circ}$ & $-2^{\circ} \pm 6^{\circ}$ \\
\hline & & $3.5 \pm 2.2$ & $186^{\circ} \pm 42^{\circ}$ & $80^{\circ} \pm 6^{\circ}$ \\
\hline \multirow[t]{3}{*}{2} & $10-60$ & $9.4 \pm 2.0$ & $131^{\circ} \pm 1^{\circ}$ & $-7^{\circ} \pm 1^{\circ}$ \\
\hline & & $5.5 \pm 2.0$ & $224^{\circ} \pm 18^{\circ}$ & $-22^{\circ} \pm 5^{\circ}$ \\
\hline & & $2.7 \pm 1.9$ & $205^{\circ} \pm 18^{\circ}$ & $66^{\circ} \pm 4^{\circ}$ \\
\hline \multirow[t]{3}{*}{3} & $50-125$ & $10.3 \pm 2.3$ & $36^{\circ} \pm 1^{\circ}$ & $4^{\circ} \pm 0^{\circ}$ \\
\hline & & $6.1 \pm 2.3$ & $127^{\circ} \pm 10^{\circ}$ & $17^{\circ} \pm 4^{\circ}$ \\
\hline & & $2.5 \pm 2.3$ & $293^{\circ} \pm 10^{\circ}$ & $73^{\circ} \pm 5^{\circ}$ \\
\hline \multirow[t]{3}{*}{$\beta \mathrm{Pic}+\mathrm{Tuc}+\mathrm{TWA}$} & & $5.6 \pm 1.7$ & $47^{\circ}$ & $34^{\circ}$ \\
\hline & & $3.5 \pm 1.7$ & $126^{\circ}$ & $-16^{\circ}$ \\
\hline & & $2.1 \pm 1.6$ & $194^{\circ}$ & $51^{\circ}$ \\
\hline
\end{tabular}

Table 3. Kinematic parameters of the Gould Belt stars calculated from Eqs. (7) with the assumed $l_{0}=128^{\circ}, R_{0}=150 \mathrm{pc}$ for group $1+2$ and $l_{o}=36^{\circ}, R_{0}=150$ pc for group 3

\begin{tabular}{c|c|c|c|c|c}
\hline Group & Age, Myr & $u_{\mathrm{G}}, v_{\mathrm{G}}, w_{\mathrm{G}}$ & $V_{\mathrm{G}}, L_{\mathrm{G}}, B_{\mathrm{G}}$ & $\omega_{\mathrm{o}}, \omega_{\mathrm{o}}^{\prime}, \omega_{\mathrm{o}}^{\prime \prime}$ & $k_{\mathrm{o}}, k_{\mathrm{o}}^{\prime}, k_{\mathrm{o}}^{\prime \prime}$ \\
\hline \multirow{2}{*}{$1+2$} & $<60$ & $1.1 \pm 0.4$ & $11.9 \pm 0.4$ & $-35.3 \pm 3.6$ & $12.1 \pm 2.7$ \\
& & $-11.8 \pm 0.4$ & $275^{\circ} \pm 2^{\circ}$ & $132.6 \pm 25.7$ & $-9.0 \pm 13.2$ \\
& & $1.3 \pm 0.3$ & $6^{\circ} \pm 1^{\circ}$ & $-292.5 \pm 87.9$ & $-47.6 \pm 29.9$ \\
$1+2$ & $<60$ & $2.3 \pm 0.3$ & $12.0 \pm 0.4$ & $-23.1 \pm 2.2$ & $14.0 \pm 2.2$ \\
& & $-11.1 \pm 0.3$ & $285^{\circ} \pm 2^{\circ}$ & $31.3 \pm 6.5$ & $-27.3 \pm 6.5$ \\
& & $1.3 \pm 0.3$ & $6^{\circ} \pm 2^{\circ}$ & - & - \\
3 & $50-125$ & $0.7 \pm 0.8$ & $11.2 \pm 0.8$ & $54.7 \pm 9.2$ & $-23.2 \pm 9.2$ \\
& & $-11.2 \pm 0.8$ & $273^{\circ} \pm 4^{\circ}$ & $-103.5 \pm 42.6$ & $123.4 \pm 42.7$ \\
& & $-0.1 \pm 0.5$ & $-1^{\circ} \pm 2^{\circ}$ & - & - \\
\hline
\end{tabular}

Note: The linear velocities $u_{\mathrm{G}}, v_{\mathrm{G}}, w_{\mathrm{G}}$, and $V_{\mathrm{G}}$ are in $\mathrm{km} \mathrm{s}{ }^{-1}$; the angular velocities $\omega_{0}$ and $k_{0}$ are in $\mathrm{km} \mathrm{s}^{-1} \mathrm{kpc}^{-1}$; the derivatives $\omega_{0}^{\prime}$ and $k_{\mathrm{o}}^{\prime}$ are in $\mathrm{km} \mathrm{s}^{-1} \mathrm{kpc}^{-2}$; and the derivatives $\omega_{\mathrm{o}}^{\prime \prime}$ and $k_{\mathrm{o}}^{\prime \prime}$ are in $\mathrm{km} \mathrm{s}^{-1} \mathrm{kpc}^{-3}$. 


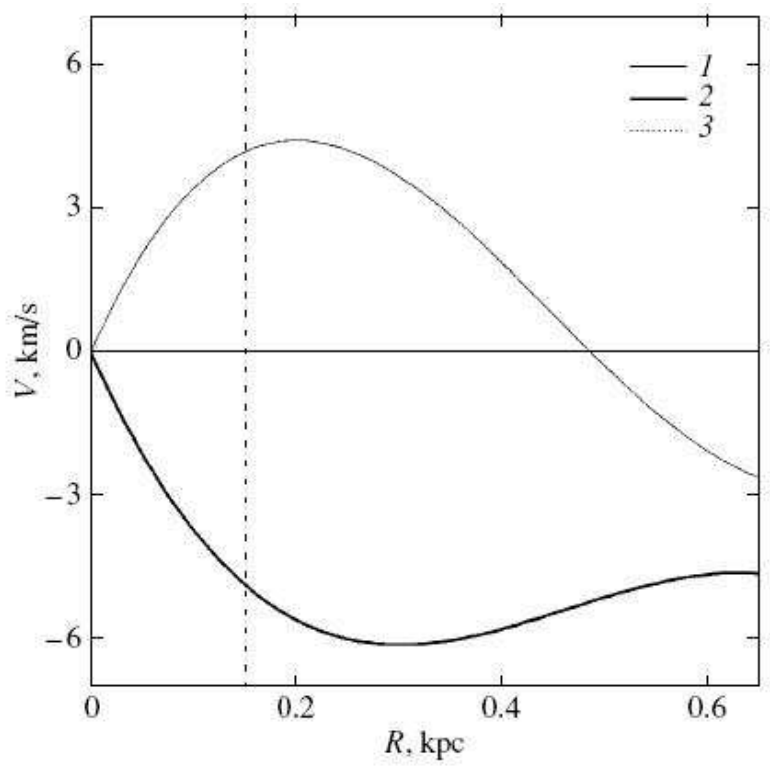

Fig. 1. Expansion ( $I$ ) and rotation (2) curves of Gould Belt stars with ages $\leq 60 \mathrm{Myr}$ (Group $1+$ Group 2); the position of the Sun is marked (3). The parameters of the curves were obtained for the center with $l_{\circ}=128^{\circ}$ and $R_{\mathrm{o}}=150 \mathrm{pc}$.

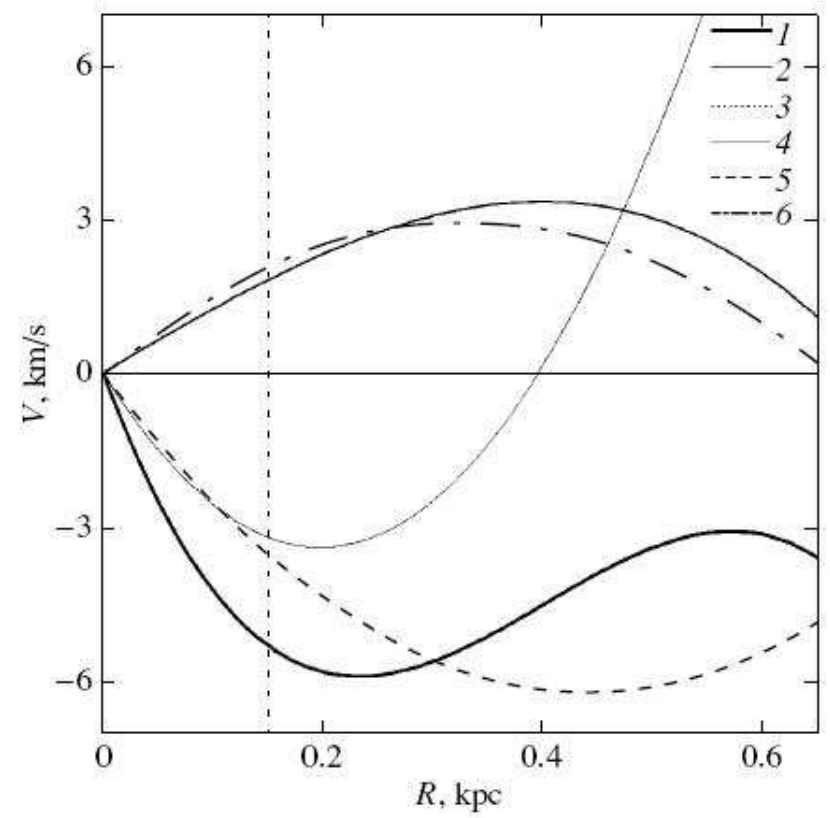

Fig. 2. Expansion $(2,6)$ and rotation $(1,4,5)$ curves of Gould Belt stars with ages $<60 \mathrm{Myr}$ (group $1+$ group 2 ); the position of the Sun is marked (3). The parameters of the curves were obtained for the center with $l_{\circ}=128^{\circ}$ and $R_{0}=150 \mathrm{pc}$. 


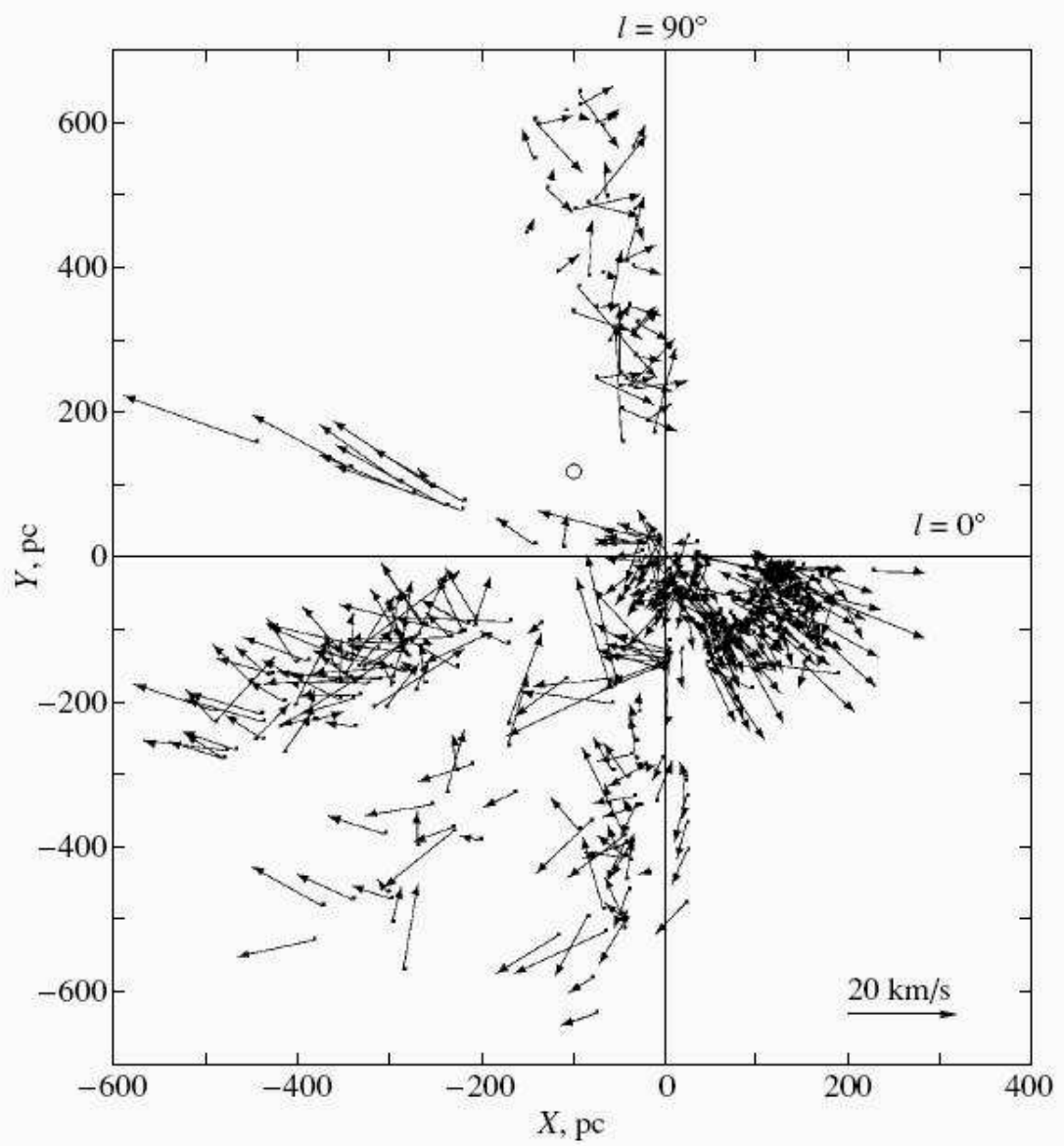

Fig. 3. Residual velocities $U$ and $V$ of Gould Belt stars with ages $<60 \mathrm{Myr}($ Group $1+$ Group 2 ) in projection onto the Galactic $X Y$ plane. The Sun is located at the coordinate origin. The circle marks the kinematic center with $l_{\mathrm{o}}=128^{\circ}$ and $R_{\mathrm{o}}=150 \mathrm{pc}$.

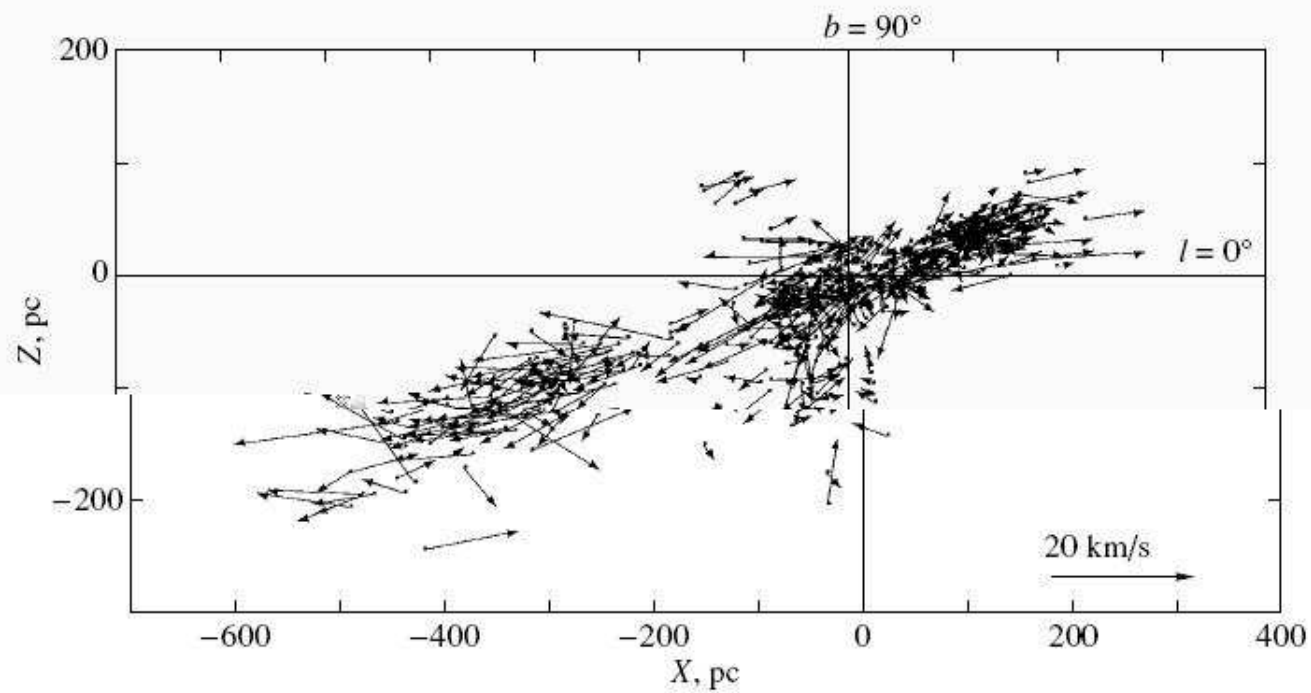

Fig. 4. Residual velocities $U$ and $W$ of Gould Belt stars with ages $<60 \mathrm{Myr}$ (Groups 1 and 2 ) in projection onto the Galactic $X Z$ plane. The Sun is located at the coordinate origin. 

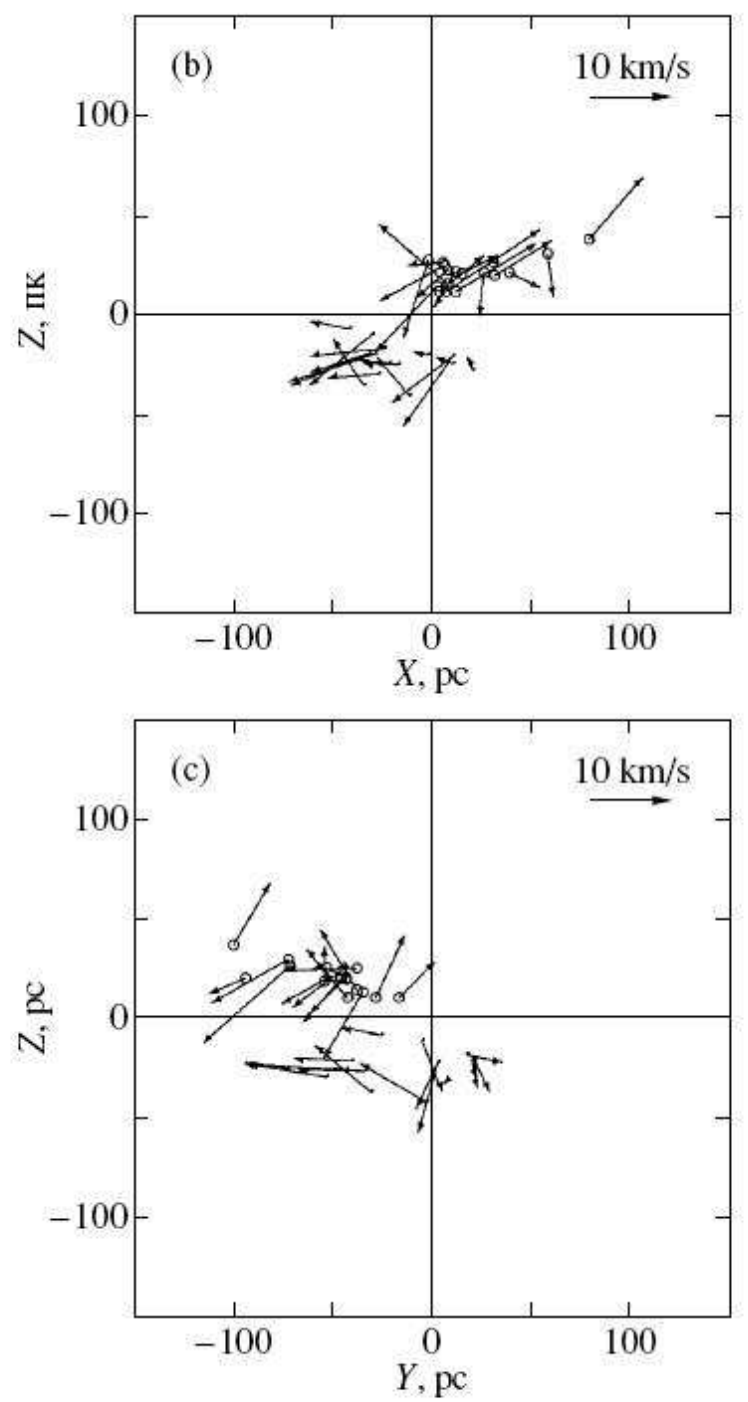

Fig. 5. Residual velocities of $\beta$ Pic and Tuc/HorA cluster stars (the positions of the stars from both groups are indicated by the circles) together with TWA cluster stars: $U$ and $V$ in projection onto the Galactic $X Y$ plane (a); $U$ and $W$ in projection onto the Galactic $X Z$ plane (b); $V$ and $W$ in projection onto the Galactic $Y Z$ plane (c). 